\title{
Foundations of a Virtual Laboratory
}

\author{
Roger Smith, Asta Richter and Ronald Ries
}

\section{Introduction}

A project was funded by the European Union to build foundations for a virtual laboratory ${ }^{1}$ where experimental and theoretical results could be combined into an audio-visual interactive presentation through the use of modern computers. The process of thin film growth and modification by energetic ion beams was chosen as the first topic for this new educational tool.

Different models for thin film growth are developed and compared to experimental results in the presentation. Particular examples include spiral formation, modelled using cellular automata, island growth using MonteCarlo methods and the first few layers of growth using classical molecular dynamics. Most of the examples are related to the growth of $\mathrm{C}_{60}$ films for which a number of experimental results had been obtained and previously reported in this journal [1]. The principle property under investigation was the structure and morphology of the films. Nine separate computer movies of the dynamics of these processes were developed on a fast PC under Windows95 and incorporated into a computer presentation made using the Authorware package. Experimental images obtained from a scanning force microscope were also included and compared to the computer models along with music and verbal explanation. In addition to the interactive version of the multi-media presentation, a non-interactive version was also made which is suitable for lectures or conference presentation. This version lasts approximately 25 minutes. Approximately 100 CD's of this work were made and are being distributed to various laboratories. A copy of the $C D$ is available on request.

\section{Software Tools}

The medium chosen for the basis of this work was the Authorware software package available from Macromedia. This software allows pictures, sounds, text and videos to be incorporated and combined together. The software allows interaction with the user and so is a very useful educational tool. It can also be developed for student learning. Educational and student assessment material can quite easily be incorporated into the package, maybe even leading to the concept of a virtual university sometime in the future. It can be especially

1 A project sponsored by the European Union: grant number ERBFMBICT 971907 useful as a research or educational tool in engineering as it also allows interactive manipulation of three dimensional structures.

The movies for the presentation consisted of a series of individual computer-generated images overlaid with sound. The three-dimensional computer generated images were produced on a UNIX workstation using the software Rayshade and POV-Ray, freely available over the internet. This software is also available for desktop computers. The input files for the image-generating software were produced from complex simulation programmes written in Fortran or Pascal. It is these programmes which contain the scientific ideas on which the simulation work is based. However in this report we do not discuss in detail the ideas behind these calculations. The movie-making techniques were discussed in a summer school held at Wildau in September 1997. A report of this summer school appears elsewhere in this volume.

The three-dimensional computer generated images were downloaded onto a PC and put together to form a movie using the package Adobe Premiere. Thus the method is similar to the way in which the first Walt Disney "Mickey Mouse" cartoons were made except that the individual frames are made by computer, not hand drawn, and the resulting movie is digitally captured on a computer, not on celluloid. Adobe Premiere is especially useful also for editing sound and editing digital movies.

\section{Examples from the presentation}

\subsection{Island growth of $\mathrm{C}_{60}$ films on glass}

Thin fullerite films were grown on glass by thermal evaporation of $\mathrm{C}_{60}$ powder. The technique by which the films were evaporated is described in detail in [2]. The images shown in figure 1 were taken using a scanning force microscope (SFM). Previous images of $\mathrm{C}_{60}$ films taken using this apparatus have been published in [1] and [2]. Figure 1 shows clear island structures which are three dimensional in nature which have distinct boundaries.

The images from the scanning force microscope can be saved in a number of different forms. For insertion into the computer presentation, the jpeg format was found to be convenient. This is a compressed image format where different compression factors can be used depending on resolution so that disc space can be optimised. 


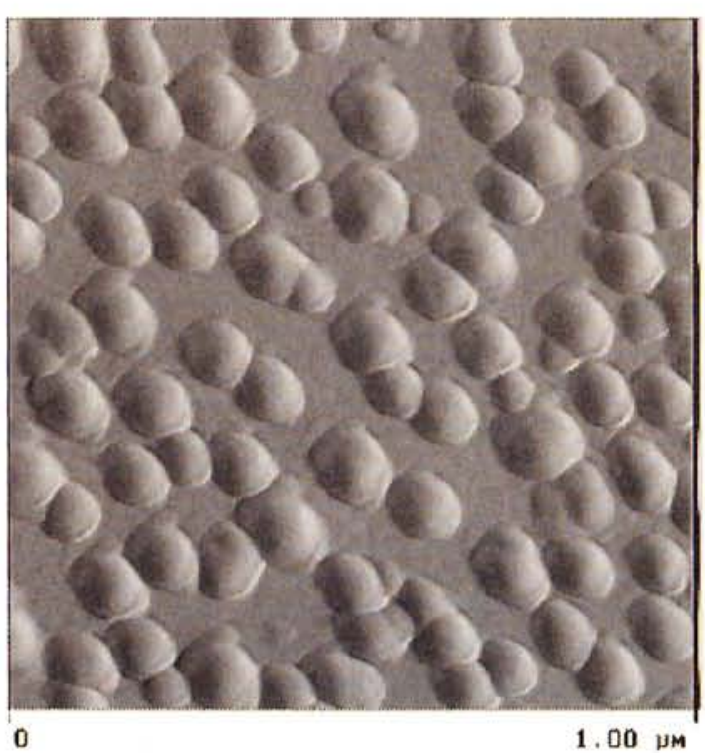

(a)

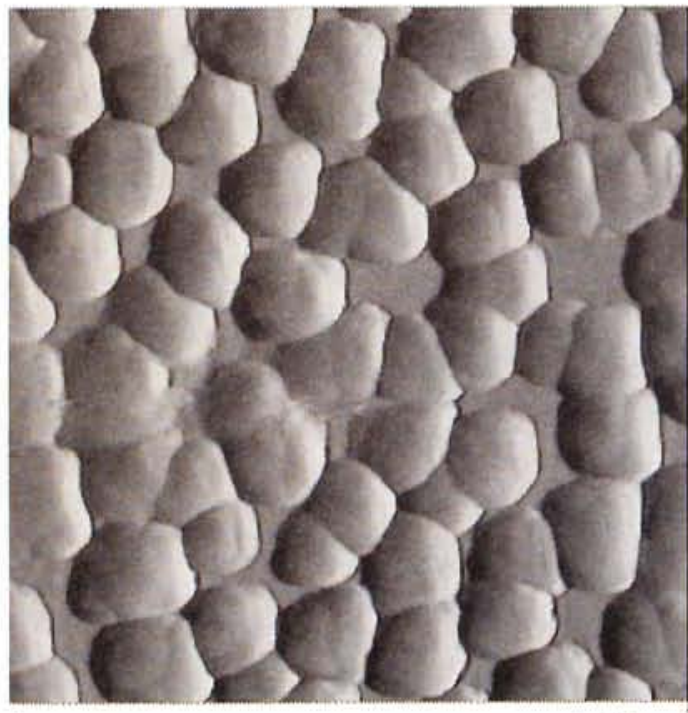

(c)

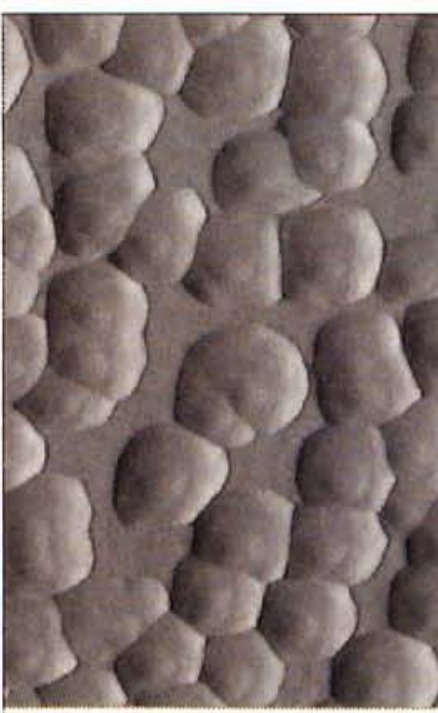

0

(b)

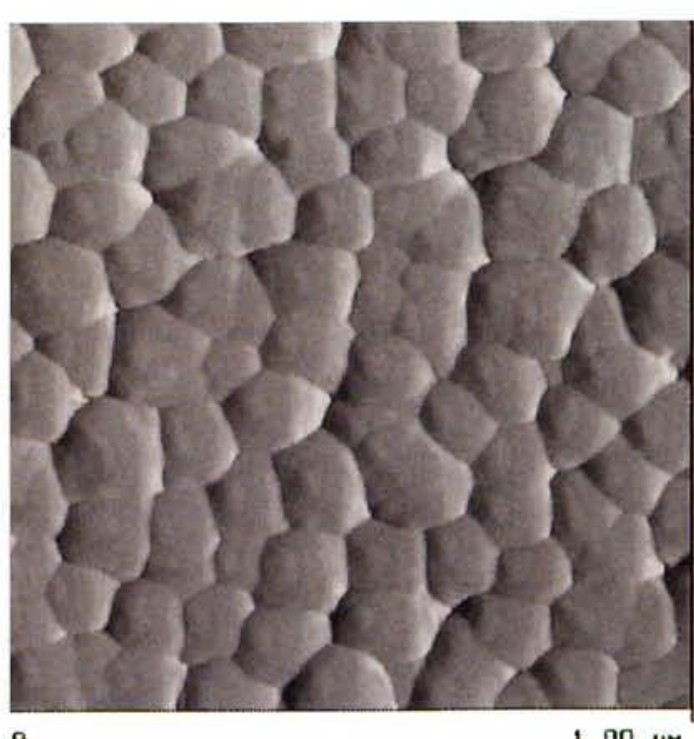

(d)

Figure 1. SFM images of $C_{60}$ films grown on glass at a temperature of $250^{\circ} \mathrm{C}$. The average film thicknesses are (a) $2 \mathrm{~nm}$, (b) $4 \mathrm{~nm}$, (c) $7 \mathrm{~nm}$, (d) $10 \mathrm{~mm}$.

This is an example of island growth. The growth model used to simulate these images is based on a Monte-Carlo scheme. Here only two-dimensional growth is considered which is an approximation used to increase the speed of the calculations compared to a full three dimensional model. The outline of the calculation is as follows: (1) The surface is assumed to be a hexagonal array of cells. (2) Particles arrive at random points on the surface and are mobile in 2-dimensions across the surface where they undertake random walks. When particles meet other particles they stick to each other to form clusters and the clusters can also move.

(3) Particles are allowed to diffuse within the cluster. There are three important parameters in the model which are the relative rates by which (a) particles arrive on the surface; (b) clusters of different sizes diffuse over the surface ; (c) particles diffuse within a cluster. If there is no diffusion within a cluster then fractal structures form such as were reported in [1] for gold deposited on ruthenium. If diffusion towards the centre of mass occurs quickly in a cluster then only circular structures appear on the surface.

We demonstrate that the island sizes depend on the diffusion rate of the clusters across the surface. In the first model we assume that the speed of the clusters is inversely proportional to the square of the number of particles $\mathrm{N}^{-2}$ in the cluster.

In the second simulation the speed is assumed to be proportional to $\mathrm{N}^{-4}$. This makes the clusters less mobile and more islands form. Figures 2 and 3 show various stages in the development of the film as it grows based on different rates by which the clusters diffuse over the surface. All other parameters are identical. In the computer presentation a dynamical version of these effects is seen.

The frame size of the movies is typically $480 \times 480$ pixels. A typical island growth movie consists of 100 frames which together with sound occupies approximately $10 \mathrm{MB}$ of disc space. 


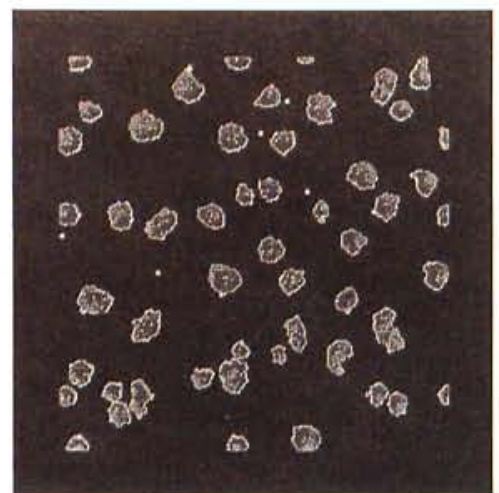

(a)

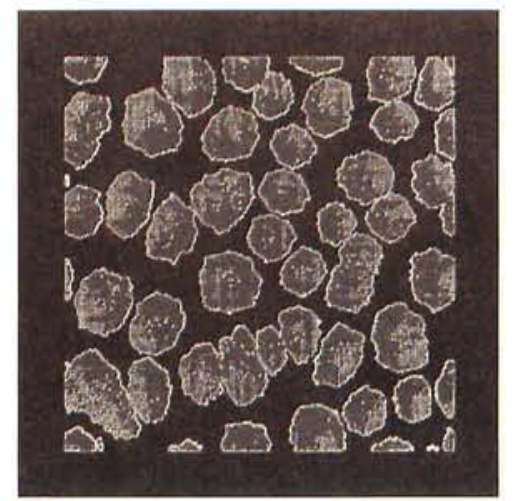

(b)

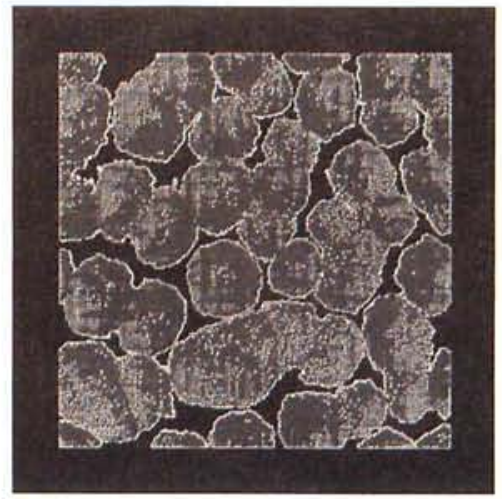

(c)

Figure 2. Various stages in the development of thin film growth using the above model with $v \sim N^{2}$. In (a) the film is only partially developed and the islands are small. In (b) the islands appear distinct and separated in (c) the film almost covers the surface.

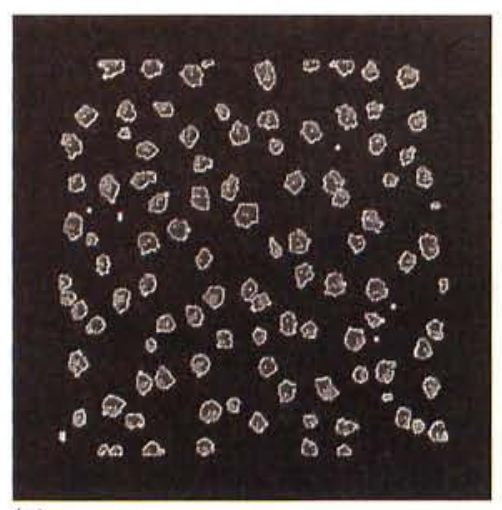

(a)

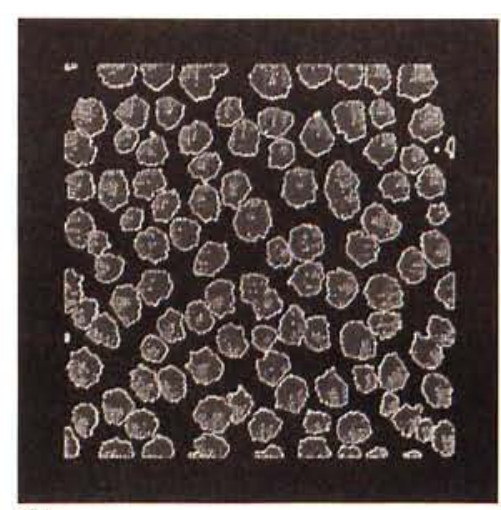

(b)

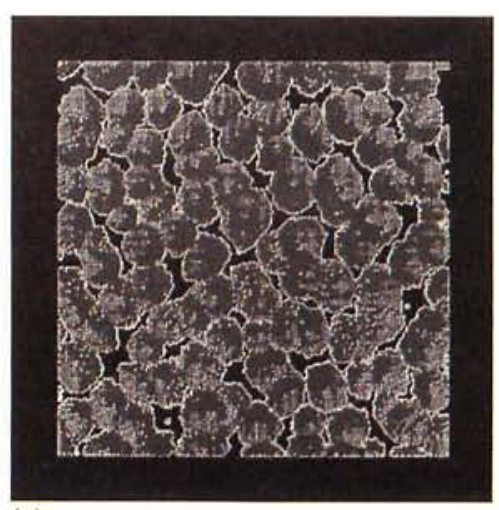

(c)

Figure 3. Various stages in the development of thin film growth using the above model with $\mathrm{v} \sim \mathrm{N}^{-1}$. The same stage of film development occurs here as in figure 2 above but the islands are smaller in size.
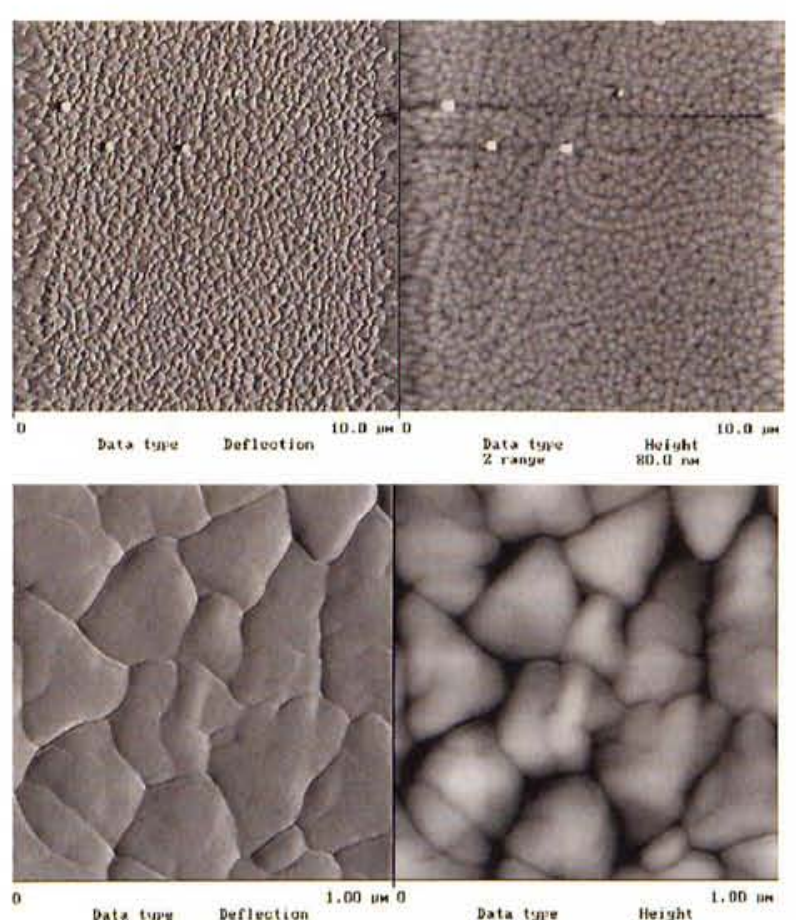

Date tyre Deflection $1.00 \mathrm{um}$

Data tow
Z range

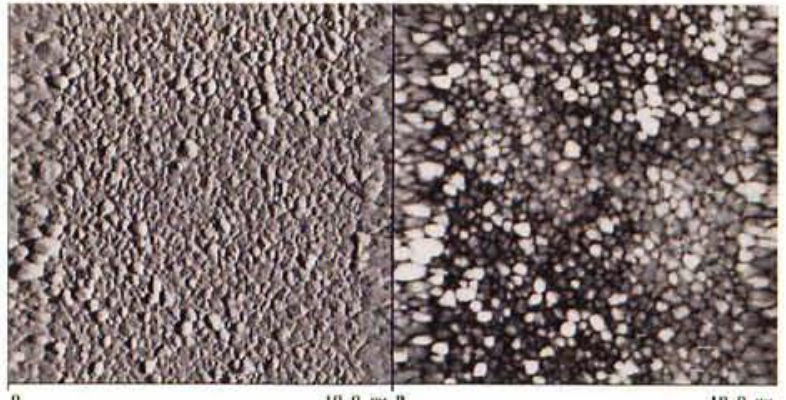

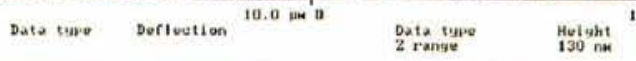

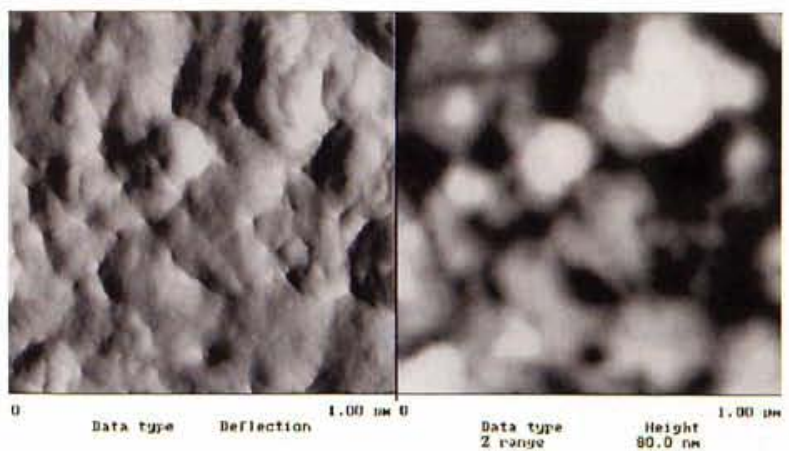

(b)

(a)

Figure 4. SFM images of different sizes, $10 \mu \mathrm{m}$ and $1 \mu \mathrm{m}$, of an $\mathrm{NaCl}$ surface after the deposition of a thin $\mathrm{C}_{60}$ film: (a) before ion bombardment and (b) after bombardment with $600 \mathrm{eV} \mathrm{Ar}{ }^{+}$ions to a dose of $10^{\prime \prime}$ ions $\mathrm{cm}^{2}$. 


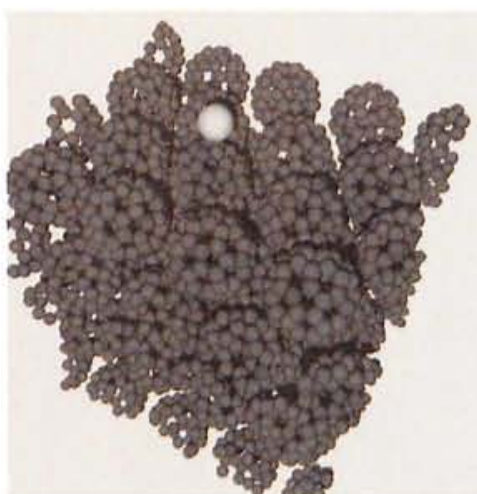

(a)

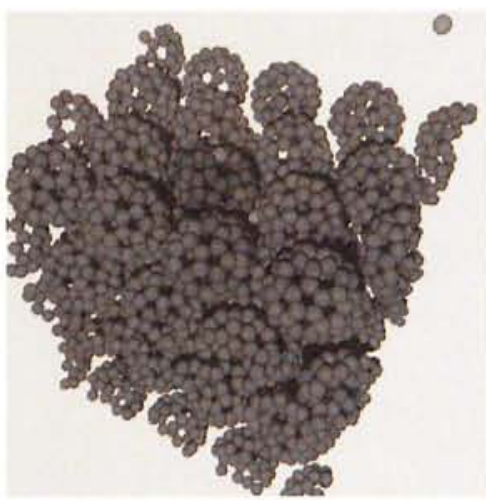

(b)

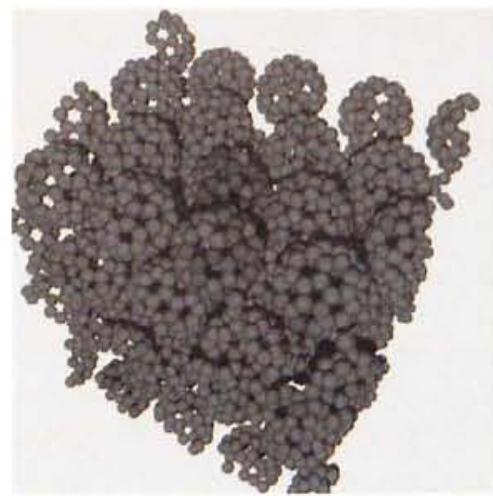

(c)

Figure 5. Molecular Dynamics computer simulation of the ion bombardment of fullerite at normal incidence and an energy of $1 \mathrm{keV}$, showing the ejection of a single $C$ atom from the surface and no other surface damage ; (a) before impact (b) $200 \mathrm{fs}$ after impact (c) 1 ps after impact.

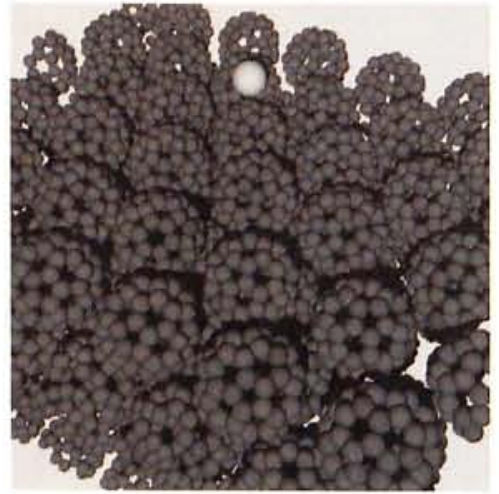

(a)

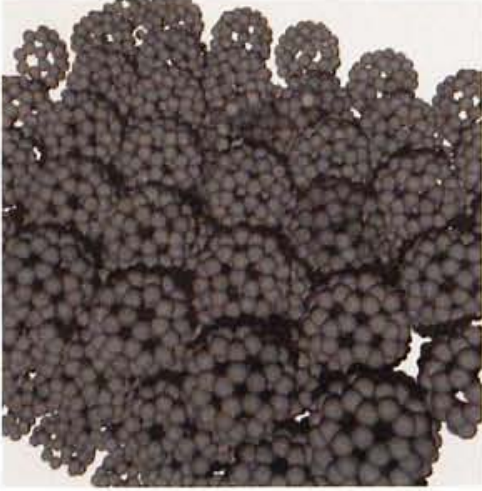

(b)

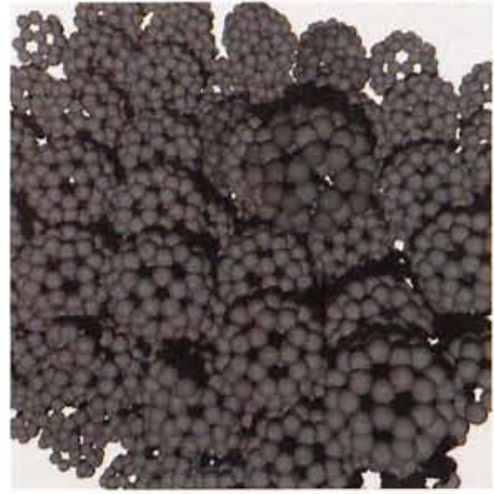

(c)

Figure 6. Molecular Dynamics computer simulation of the ion bombardment of fullerite at normal incidence and an energy of $1 \mathrm{keV}$, showing the ejection of a complete $C_{60}$ molecule from the surface and some residual polymerisation and surface damage; (a) before impact (b) 1 ps after impact (c) 5 ps after impact.

\subsection{Sputtering of fullerite films}

We demonstrate the power of the SFM combined with computer simulation for the case of the modification of fullerite by energetic ion bombardment [3]. The $\mathrm{C}_{60}$ film is deposited on $\mathrm{NaCl}$ by evaporation as in the case of deposition on glass. A freshly cleaved $\mathrm{NaCl}$ crystal consists of spirals and terraces over the scale of about $10 \mu \mathrm{m}$. Deposition of $\mathrm{C}_{60}$ molecules on the $\mathrm{NaCl}$ surface results in decoration of the terraces as can be seen in the unbombarded film in figure 4 . Figure 4 contains SFM images of the film both before and after bombardment with energetic ions. Figure 4 also shows that crystal grains have formed and these are limited in size by the size of the terrace edges. Over a smaller scale of $1 \mu \mathrm{m}$ the films appear reasonably flat but with distinct grain boundaries visible. The ion bombarded $\mathrm{C}_{60}$ film is approximately 4 times rougher than the unbombarded surface and the grain boundaries are no longer well defined. It is found that there is partly a decomposition of the $\mathrm{C}_{60}$ material into an amorphous carbon structure.

The simulation technique used for the modelling is classical molecular dynamics (MD). The technique is described in detail in [4]. This book describes many applications of MD in surface science studies. The simulation identifies different mechanisms by which the surface can be damaged as a result of momentum transfer. (In this simulation we do not consider electronic stopping). Individual carbon atoms can be ejected from the crystal by direct collisional means. Such an event is described in figure 5 . In this case the surface remains largely intact as a result of this one collision.

In addition to single particle ejection, full $\mathrm{C}_{60}$ molecules can also be sputtered intact. Such a case is described in figure 6 . In this case more energy is deposited near the surface. The molecule is desorbed from the surface over a longer timescale than the individual carbon atom was ejected, of the order of picoseconds $\left(10^{-12} \mathrm{~s}\right)$ rather than femtoseconds $\left(10^{-15} \mathrm{~s}\right)$. In this second case more surface damage occurs. Other cases can show dimer ejection or no surface damage whatsoever if the ion is incident in a channelling direction. It just depends on the impact point on the surface.

Computer movies of the events described in figures 5 and 6 have also been made and incorporated into our presentation. These movies consist of more frames than the Monte-Carlo simulations described above. Typically they contain 1000 frames where each frame is a jpeg image of the order of $60 \mathrm{~KB}$ in size. Together with sound such a movie could require up to $80 \mathrm{MB}$ of disc space! Thus movies are expensive in storage and require a fast computer to run well. Our full presentation takes approximately $500 \mathrm{MB}$ of space and a Pentium II PC with a 32 MB RAM, running Windows95 with good graphic and sound cards is a minimum requirement. 


\section{Conclusion}

As computers become faster and smaller, the use of interactive audio visual techniques as a presentational medium will increase. The development of a single package on the topic of Thin Film Growth does not constitute a virtual laboratory as such, but it is possible to envisage a time in the future where much educational and research material is presented through the computational medium. The ability for students to interact with such presentations means that assessment as well as learning tasks, for example by trial and error, are possible from a computer terminal. It has the advantage that equipment is not broken if a mistake is made.

We have not in this presentation tried to simulate a particular experiment. This is also possible within the context of the new medium but requires a very large amount of programming work and considerable skill. What we have done instead is to combine the results of experiments together with computer simulations to help with scientific understanding in such a way that the user can access the material in a similar way to reading a book.

\section{References}

[1] A. Richter and R. Smith, Wissenschaftliche Beiträge der TFH Wildau 721 (1996)

[2] A. Richter, R. Ries, K. Szulzewsky, B. Pietzak and R. Smith, Surface Science 201394 (1997)

[3] S. Hobday, R. Smith, U. Gibson and A. Richter, Radiation Effects and Defects in Solids 310142 (1997)

[4] R. Smith Atom and Ion Collisions in Solids and at Surfaces, CUP, Cambridge, UK (1997)

\section{Authors}

\section{Prof. Dr. Roger Smith}

Loughborough University

Mathematical Sciences Department

Loughborough, LE11 3TU, UK

E-mail: R.Smith@lboro.ac.uk

\section{Prof. Dr. Asta Richter}

\section{Dipl.-Kristallogr. Ronald Ries}

Technische Fachhochschule Wildau

Fachbereich Ingenieurwesen/Wirtschaftsingenieurwesen

Tel. (0 33 75) 507-219

E-mail: richter@pt.tfh-wildau.de 